\title{
Values-Focused Exposure and Response Prevention in the Treatment of Comorbid Schizophrenia and Obsessive-Compulsive Disorder: The Case of "Mr. H"
}

\author{
ASHLEIGH GOLDEN ${ }^{\mathrm{a}, \mathrm{c}}$ \& ROBERT M. HOLAWAY ${ }^{\mathrm{b}}$ \\ ${ }^{a}$ PGSP-Stanford Psy.D. Consortium and Student Health \& Counseling Services, University of California, Davis \\ b Pacific Anxiety Group, Menlo Park, CA, and PGSP-Stanford Psy.D. Consortium \\ C Correspondence regarding this article should be addressed to Ashleigh Golden, Student Health and Counseling \\ Services, University of California—Davis, 219 North Hall, One Shields Avenue, Davis, CA 95616 \\ Email: asgpsyd@stanford.edu
}

\begin{abstract}
We describe a subgroup of individuals with comorbid diagnoses of paranoid schizophrenia and obsessive-compulsive (OCD) disorder. It is proposed that traditional exposure and response prevention (ERP) may need to be altered given the unique characteristics of this subgroup. We suggest that providers may approach treatment for OCD from within the context of a patient's delusion. The case study of "Mr. H," a veteran of the U.S. military diagnosed with schizophrenia and OCD, is described. We propose that attenuating or eliminating obsessions and compulsions may not result in the achievement of a goal stemming from a delusional belief, but may contribute to an increased sense of mastery and independence.
\end{abstract}

Key words: obsessive compulsive disorder (OCD); exposure and response prevention (ERP); cognitivebehavior therapy (CBT); paranoid schizophrenia; delusion; comorbidity; case study; clinical case study

\section{CASE CONTEXT AND METHOD}

The following case study of "Mr. $\mathrm{H}$ " was selected for the successful outcome resulting from the application of a novel conceptualization in the treatment of obsessive-compulsive disorder (OCD) co-occurring with schizophrenia. Recognizing the complex nature of the task and the limited research on previous treatments in similar cases, the treatment team chose to supplement the standard exposure and response prevention (ERP) method for treating OCD with a values-based focus.

Mr. $\mathrm{H}$ was referred to the treatment team by another psychologist at the clinic only for the treatment of his obsessive-compulsive symptoms, not his delusions. Prior to Mr. H's current referring psychologist, other mental health care providers responsible for his care had not attempted to institute referrals for the implementation of ERP with Mr. $\mathrm{H}$ because they assumed that his delusions would need to be treated successfully before his being able to complete an effective round of ERP. 
Values-Focused Exposure and Response Prevention in the Treatment of Comorbid

Schizophrenia and Obsessive-Compulsive Disorder: The Case of "Mr. H"

A. Golden and R. M. Holaway

Pragmatic Case Studies in Psychotherapy, http://pcsp.libraries.rutgers.edu

Volume 9, Module 1, Article 3, pp. 65-80, 02-17-13 [copyright by authors]

Mr. H was seen over a course of twelve sessions by the first author, a doctoral candidate in clinical psychology, who was supervised by the second author, a licensed psychologist in a Veterans Affairs (VA) outpatient clinic setting. The therapist met with her supervisor once per week for the duration of Mr. H's treatment. Initial meetings focused on the discussion of case conceptualization and treatment planning, while remaining meetings served to verify that the therapist was adhering to the original treatment plan and to monitor the efficacy of the treatment. Throughout the course of psychotherapy, Mr. H's medication was managed regularly by a psychiatrist, another member of the treatment team with whom the therapist and her supervisor consulted for input into the case. The therapist had regular contact with the Mr. H's board-andcare operator, Ms. O, who often accompanied Mr. $\mathrm{H}$ to treatment and requested one session with the therapist to update her on Mr. H's progress. The therapist sought Mr. H's consent to maintain regular telephone and in-person contact Ms. O. Through an electronic database, the treatment team was also able to access Mr. H's prior treatment reports and medical records to inform conceptualization and treatment planning.

\section{THE CLIENT}

Mr. H was a 47-year-old, White, single, male veteran of the U.S. military diagnosed with comorbid OCD and paranoid schizophrenia, who lived in a board-and-care home. Mr. $\mathrm{H}$ had been participating in an ongoing day treatment program for individuals with severe mental illness for the previous 5 years with the intention of managing his schizophrenia, which had long been considered the more debilitating diagnosis of the two by his mental health care providers.

Mr. H continued to express delusions of persecution and grandeur, although he was stabilized on antipsychotic medication by means of regular meetings with his treating psychiatrist. Mr. H expressed beliefs that the FBI had implanted a machine in his brain and that famous movie directors had filmed him in the past. He regularly reported that he was destined for a career as a Hollywood movie star and would require the help of relationships with two well-known movie actresses to follow his calling.

However, Mr. H believed that to fulfill this destiny, he must first become "normal;" that is, he must first become free of compulsive hand-washing behaviors. With regard to obsessions, Mr. H expressed fears of becoming contaminated from contact with floor mops, indoor flooring, toilet seats without paper covers, and other "dirty” surfaces. His compulsions primarily included washing his hands with soap and water or spitting on them when no water was available. Mr. $\mathrm{H}$ was able to acknowledge that his "mind" was telling him that the surfaces were dirty when they were in fact clean. Mr. H was referred to an outpatient mental health clinic by his psychologist to specifically address his obsessions and compulsions. 
Values-Focused Exposure and Response Prevention in the Treatment of Comorbid

\section{GUIDING CONCEPTION WITH RESEARCH AND CLINICAL EXPERIENCE SUPPORT}

\section{Exposure and Response Prevention}

The therapist and the supervising psychologist both had experience in treating patients with OCD using ERP, as well as psychotic disorders by means of cognitive-behavioral therapy; however, neither had prior experience treating OCD as a primary concern for an individual contending simultaneously with schizophrenia. Both clinicians had experience in treating individuals suffering from a variety of anxiety disorders, including OCD, social anxiety disorder, generalized anxiety disorder, post-traumatic stress disorder, and specific phobias.

Both the therapist and her supervisor were very familiar with the idea that ERP is considered the "gold standard" and essential treatment for addressing OCD, understanding that implementing exposure exercises with the patient and helping the patient to engage in response prevention were necessary components of the treatment (Foa, Steketee, Grayson, Turner, \& Latimer, 1984). Exposure and response prevention entails patients willingly exposing themselves to feared stimuli, real or imagined, while refraining from performing their compulsions or other neutralizing behaviors (Kozak \& Foa, 1997). The idea is that as patients expose themselves to feared stimuli and refrain from neutralizing behaviors, they habituate to the associated anxiety and learn to increase their tolerance for uncertainty and risk, contributing to decreased distress and behavioral avoidance over time.

As with any behavioral treatment, the clinical team worked through the stages of assessment, case conceptualization and treatment planning, and monitoring of the efficacy of the therapy throughout the course of treatment. Since the clinical picture of OCD in this case was complicated by a diagnosis of schizophrenia, the treatment team consulted the research literature for guidance concerning the treatment of patients with comorbid OCD and psychosis. It was discovered that approximately 8-26\% of patients with schizophrenia meet DSM-IV criteria for OCD (Owashi, Ota, Otsubo, Susa, \& Kamijima, 2010). According to the National Comorbidity Survey (2005), the lifetime prevalence of OCD is 2.7\%, while that of schizophrenia is estimated to be $0.5-1.5 \%$ (APA, 1994).

Schizophrenia with obsessive-compulsive symptoms (OCS) or full obsessive compulsive disorder (OCD) displays unique clinical characteristics not typically observed when assessing patients with only one of the disorders (Poyurovsky, Weizman, \& Weizman, 2004). Hwang, Morgan, and Losconzcy (2000) found that patients with "obsessive-compulsive-schizophrenia” demonstrate a distinct clinical and neuropsychological profile on the schizophrenic spectrum, such that it is unclear whether to classify the subgroup as representing a schizophrenic subtype with obsessive-compulsive symptoms or a subgroup of individuals with comorbid schizophrenia and OCD. In either case, the authors recognize that such a diagnostic profile necessitates special treatment strategies that adequately address both clinical phenomena and ensure the best possible treatment outcome. 
Values-Focused Exposure and Response Prevention in the Treatment of Comorbid

Schizophrenia and Obsessive-Compulsive Disorder: The Case of "Mr. H"

A. Golden and R. M. Holaway

Pragmatic Case Studies in Psychotherapy, http://pcsp.libraries.rutgers.edu

Volume 9, Module 1, Article 3, pp. 65-80, 02-17-13 [copyright by authors]

Owashi et al. (2010) examined patients with schizophrenia in an inpatient unit exhibiting OCD or OCS. The authors found that the patients with comorbid schizophrenia and OCD displayed more severe negative symptoms, including emotional withdrawal and depressed mood, compared to patients with comorbid schizophrenia and OCS. The patients with comorbid schizophrenia and OCD demonstrated more feelings of guilt, somatic concern, and depression compared to patients with schizophrenia without comorbid OCD. Patients with comorbid schizophrenia and OCS exhibited more hallucinations, unusual thought disorder, exaggerated self-esteem, and somatic concern than patients with schizophrenia without comorbid OCS.

Confusing the diagnostic question further is the DSM-IV specifier for OCD, "with poor insight," as it does not address whether a patient with OCD who lacks insight regarding the nature of his/her obsessive beliefs or fears should be diagnosed with a psychotic disorder rather than OCD (Leckman et al., 2010). The DSM-IV field trial of OCD (Foa et al., 1995) revealed that $4 \%$ of patients with OCD had no insight and $26 \%$ had little insight into the excessiveness or irrationality of their obsessions and/or compulsions. Thus, OCD can be characterized by a continuum of insight that includes delusional beliefs (Leckman et al., 2010).

(Note that Leckman and colleagues [2010] therefore suggest an expansion of the specifier that includes a category for delusional OCD beliefs, indicating that an individual is completely convinced that the OCD beliefs are accurate. Thus, if this is the case, where does OCD end and schizophrenia begin? Leckman et al. [2010] observe that the definition of a delusion can be equally applied to a schizophrenic delusion as well as an obsessive-compulsive delusion in an individual who lacks insight. On the other hand, bizarre somatic cognitions or those that demonstrate a loss of control over mind or body are more prevalent in schizophrenia than in OCD with poor insight [APA, 1994]).

With respect to pharmacological treatment, research estimates that $30-40 \%$ of clients with schizophrenia in the U.S. are prescribed combinations of two or more antipsychotic medications (Clark, Bartels, Mellman, \& Peacock, 2002; Covell, Jackson, Evans, \& Essock, 2002; Noordsy, Mellman, Larsen, Logthetis, \& O’Keefe, 2001). Some researchers have found success in and recommend treating comorbid schizophrenia and OCD with a similar combination of pharmacological treatments. Examples are clozapine and paroexetine (Srous, Patel, Zimmet, \& Alan, 1999); a second-generation antipsychotic and a serotonin reuptake inhibitor (Poyurovsky, Weizman, \& Weizman, 2004; American Psychiatric Association, 2007); or antipsychotics and an adjunctive selective serotonin reuptake inhibitor or clomipramine (Hwang, Yum, Kwon, \& Opler, 2005). McDonough and Kennedy (2002) discuss the use of antipsychotics with patients with OCD with poor insight, delusions, or schizophrenia. "Antipsychotics, although they can be tried, are not the mainstay of treatment for patients with this delusional variant, since behavior therapy and/or SRIs are often effective alone” (McDonough \& Kennedy, 2002, p. 131).

With regard to psychological treatment, the cognitive-behavioral approach of ERP is clearly efficacious for OCD (van Balkom et al., 1994; Abramowitz, 1997); however, its efficacy for OCD with comorbid schizophrenia is not yet known (Rodriguez, Corcoran, \& Simpson, 2010). Interestingly, Foa and Kozak (1994) found that OCD with poor insight did not predict poor outcomes for behavioral treatment in the majority of studies. It is, however, important to 
Values-Focused Exposure and Response Prevention in the Treatment of Comorbid

Schizophrenia and Obsessive-Compulsive Disorder: The Case of "Mr. H"

A. Golden and R.M. Holaway

Pragmatic Case Studies in Psychotherapy, http://pcsp.libraries.rutgers.edu

Volume 9, Module 1, Article 3, pp. 65-80, 02-17-13 [copyright by authors]

note that patients with extremely strong (fixed) beliefs in their obsessive ideation may demonstrate poor outcomes (Foa, Abramowitz, Franklin, \& Kozak, 1999). Foa and Franklin (2008) suggest that treatment compliance may be an important factor in the lack of response to treatment observed in individuals with over-valued ideation. They further hypothesize that treatment compliance may mediate the relationship between fixed beliefs and treatment outcome.

To specifically target the obsessive-compulsive symptoms in comorbid schizophrenia and OCD, Rodriguez and colleagues (2010) remark that the two first-line treatments are serotonin reuptake inhibitors and ERP. Rodriguez and colleagues (2010) discussed the course of combination pharmacological treatment for a 19-year-old man, “Carlos," diagnosed with schizophrenia in addition to OCD. The authors mention that ERP was implemented as an adjunctive treatment to address residual obsessive-compulsive symptoms after trials of serotonin reuptake inhibitors had been attempted and Carlos was stable on an outpatient regimen of medications. Although Carlos participated in a trial of ERP for his residual obsessivecompulsive symptoms as planned, he ultimately chose to drop out of therapy, as he had little confidence that it would work. Carlos continued to experience impairment after dropping out of treatment with ERP, leading Rodriguez and colleagues (2010) to question, "Given the efficacy of exposure and response prevention for OCD, is it a safe and effective option for patients with comorbid schizophrenia and OCD who are stabilized on antipsychotics?” (p. 759).

Ganesan, Kumar, and Khanna (2001) studied a group of fifteen patients with OCD and psychosis, ultimately advocating for the use of a combination treatment of anti-obsessional and antipsychotic medications. However, they also report that ERP was attempted in three patients with mixed results: The introduction of ERP corresponded with an exacerbation of psychotic symptoms in one patient, whereas an improvement in obsessive-compulsive symptoms was observed in the two other patients.

\section{Acceptance and Commitment Therapy (ACT)}

Acceptance and Commitment Therapy (ACT) employs acceptance and mindfulness practices to offer alternative ways of relating to unwanted internal experiences, emphasizing a valued-based approach, that is, an approach in which valued-action is viewed as integral to living a meaningful life (Hayes, Strosahl, \& Wilson, 1999). ACT argues that directly challenging psychotic symptoms may produce paradoxical rebound effects by exacerbating entanglement with odd cognitions (Pankey, 2003; Bach and Hayes, 2002). Indeed, mental health professionals who directly challenge psychotic symptoms can inadvertently contribute to highly negative experiences for individuals with psychosis (Bloy, Oliver, \& Morris, 2011). Suppressing psychotic symptoms can actually increase the frequency of unwanted thoughts (Salkovskis \& Campbell, 1994; Wegner, Schneider, Carter, \& White, 1987).

Among other foci, ACT concentrates on behavior that may help an individual travel further in his or her valued directions even in the midst of difficult experiences (Pankey, 2003). One of the goals with a psychotic population is to help the client accept a difficult psychological context (such as experiencing some disturbance from psychotic symptoms) while simultaneously concentrating on valued overt behavior change (Pankey, 2003). ACT thus "seeks to increase 
clients' willingness to be exposed to unpleasant private events if necessary to complete valued activities" (Hayes, Strosahl, \& Wilson, 1999, p. 81). ACT research on psychotic populations is limited but promising (Garcia \& Ferez, 2001; Bach \& Hayes, 2002; Gaudiano \& Herbert, 2006), with recent studies suggesting that ACT may promote recovery by reducing rates of relapse (Bach \& Hayes, 2002; Gaudiano \& Herbert, 2006). Bloy, Oliver, and Morris (2011) describe the use of ACT with an individual having experienced longstanding psychosis, including paranoia and delusions. While the individual did not completely recover from his psychotic symptoms, he reported that he continued to engage in valued activities and experienced an improvement in quality of life despite ongoing distress and psychotic symptoms.

\section{ASSESSMENT OF THE CLIENT'S PROBLEMS, GOALS, STRENGTHS, AND HISTORY}

As noted above, Mr. H suffered from contamination obsessions including fear of contact with mops, floors, toilet seats, and other "dirty" surfaces. After coming into contact with such "contaminated" surfaces, his ritualistic behaviors included washing his hands with soap or water or spitting on them if there was no faucet in the immediate vicinity. He stated that when he was three years-old, his mother performed sexual acts with men in plain sight, which he found to be "disgusting” and may have been associated with the eventual onset of his obsessions. He reported that he first began to notice a need to hand-wash in the 1970s, when he believed that a famous movie director was filming him through a window in his apartment as he snorted cocaine off the floor. However, the tangential and somewhat non-sequitur nature of Mr. H's speech made the therapist cautious about the accuracy of the Mr. H's historical narrative.

Mr. $\mathrm{H}$ also demonstrated delusional beliefs, including the ideas that he had been filmed by well-known movie directors in the past, that the FBI had installed a machine in his brain, and that his destiny was to become a movie star in Hollywood through the help of relationships with two movie actresses.

During his initial interview, Mr. H reported that being "normal” (i.e., free of timeconsuming hand-washing behaviors) was more important in itself than serving as a means of helping him to establish relationships with famous women and become a movie star. Thus, according to Mr. H, his compulsions were more distressing than his delusional beliefs, and learning to control them was more important than working to become more in touch with reality.

Mr. H lived in a board-and-care facility with several other residents under the direction of a female operator, supervisor, Ms. O. After obtaining consent from Mr. H, the therapist contacted Ms. O for additional information regarding Mr. H's living situation and current functioning. Ms. $\mathrm{O}$ reported that Mr. H lined up for his board-and-care home's restroom faucet every half hour and demanded that his walker be washed upon returning to the board-and-care facility from appointments or meetings. Ms. O said that she often took the residents on outings in a van, and that Mr. H ordered that she stop the van frequently at rest stops so that he might locate a faucet and wash his hands. Ms. O described Mr. $\mathrm{H}$ as being debilitated by his contamination obsessions and perceived need to wash his hands; the debilitating effects included abundant time spent 
Values-Focused Exposure and Response Prevention in the Treatment of Comorbid

Schizophrenia and Obsessive-Compulsive Disorder: The Case of "Mr. H"

A. Golden and R. M. Holaway

Pragmatic Case Studies in Psychotherapy, http://pcsp.libraries.rutgers.edu

Volume 9, Module 1, Article 3, pp. 65-80, 02-17-13 [copyright by authors]

waiting to wash his hands throughout the day and disrupting other residents with his need to occupy the home's bathroom and make frequent stops on outings.

Mr. H was motivated by the level of impairment that he was experiencing to follow through with the referral from his psychologist as well as the insight that his obsessions were in fact excessive and unreasonable. He had a gentle, kind demeanor that seemed to lend itself to the collaborative nature of the therapy, such as the development of the fear hierarchy and the carrying out of the exposures themselves.

\section{FORMULATION AND TREATMENT PLAN}

Due to Mr. H's questionable abilities as a historian, it is unknown whether he began to experience obsessive and/or compulsive symptoms as a child after watching his mother engage in sexual acts with men, after believing that he had been witnessed snorting cocaine off the floor in his apartment by a movie director, after another incident, or through a more gradual development. In any event, the maintenance of Mr. H's obsessions and compulsions was cyclical in nature. He encountered surfaces which he believed to be contaminated. He then experienced anxiety and an urge to engage in his ritualistic hand-washing or spitting behaviors. Succumbing to this coping behavior for his anxiety (a) prevented him from habituating to his anxiety, (b) interfered with an ability to acquire new strategies to cope with his obsessive fears, and (c) heightened the power of his urges, increasing his sense of their necessity and irresistibility. Mr. H's compulsive hand-washing was his strategy to attempt to minimize or avoid the anxiety that he experienced after having come into contact with supposedly contaminated surfaces. The more he engaged in hand-washing as a means to deal with his anxiety, the more natural, necessary, and irresistible it felt. Therefore, the more he experienced anxiety about contaminated surfaces, the more he employed this strategy, maintaining a cycle of behavior.

The treatment team decided to implement a course of ERP to address Mr. H's compulsive hand-washing. Implementation of ERP was tailored to Mr. H's clinical presentation, taking into account his positive psychotic symptoms. As a result, it was decided to attempt to treat his obsessions and compulsions within the context of his delusions. In this way, the treatment plan combined a values-based approach, derived from ACT theory as described above in the Guiding Conception section, with the standard ERP approach for OCD.

Mr. H exhibited chronic delusions of grandeur to which he was deeply attached. He had a long history of traditional psychosocial therapy for psychosis and medication management by a psychiatrist with little improvement in his psychotic delusions. He also had been referred to the treatment team by another psychologist for his obsessions and compulsions, rather than his delusions themselves. The treatment team additionally recognized that his obsessions and compulsions comprised the main source of his distress. The treatment team hypothesized that an ACT-derived, values-based model might enhance the patient's ERP treatment. Though he might continue to experience some distress from his psychotic symptoms, it was believed that this approach would help him to move in his valued direction by changing behaviors surrounding his compulsions. Thus, in line with ACT theory, his delusional beliefs were reframed as his life values. If $\mathrm{Mr} . \mathrm{H}$ and the treatment team were successful in collaborating to reduce the frequency 
Schizophrenia and Obsessive-Compulsive Disorder: The Case of "Mr. H"

A. Golden and R.M. Holaway

Pragmatic Case Studies in Psychotherapy, http://pcsp.libraries.rutgers.edu

Volume 9, Module 1, Article 3, pp. 65-80, 02-17-13 [copyright by authors]

of his compulsive behaviors - if he became "normal," in his words - then it was expected that his quality of life would improve, even if he did not reach his ultimate goal of becoming a Hollywood star (or eradicate his delusional beliefs).

\section{COURSE OF THERAPY}

Mr. H suffered from mobility issues and used a walker to navigate his environment. At the beginning of the first session, Mr. H expressed his enjoyment of coffee, particularly that which the VA outpatient clinic provided via a drip coffee machine located in the lobby. Mr. $\mathrm{H}$ explained that it was difficult for him to hold onto his walker while pouring himself coffee, and would be greatly appreciative if I as the the therapist could perform this action for him. As an alliance-building behavior, I heeded his request, and also asked him at the beginning of every subsequent session whether he would care for a cup of coffee; $\mathrm{Mr}$. $\mathrm{H}$ always replied in the affirmative and expressed his gratitude. I also kept a bowl of candies for patients on the desk in my office. During his first visit, Mr. H glanced repeatedly at the dish and eventually asked for some candies. During every subsequent session, I inquired whether Mr. H would care for some candies; he always took a handful. I hoped that my attendance to Mr. H's wishes would serve as an alliance-building strategy that might engender trust and willingness to work with me through difficult exposure activities. Several times after my fetching coffee for Mr. H, he called me "a very nice lady.”

During the first session, which involved an assessment of Mr. H's presenting complaints, Mr. H explained the nature of his obsessions and compulsions, as well as his distress surrounding these behaviors. I listened actively, making empathic restatements and reflections as $\mathrm{Mr} . \mathrm{H}$ explained his concern that "normal people" did not have obsessive worry or engage in compulsive behaviors, and that he would never be able to maintain relationships with famous women in order to become a movie star if he continued to compulsively hand-wash.

The second session involved psychoeducation regarding exposure and response prevention for compulsive behavior. Every session with $\mathrm{Mr}$. H began with a reiteration of the rationale behind the treatment, which he claimed that he sometimes forgot.

The third session was comprised of the development of a fear hierarchy. I explained the concept of subjective units of distress (SUDS) using a scale of 1-10. Mr. H and I brainstormed situations that triggered fear of contamination for the patient. Mr. H expressed that each situation would be equally "impossible." The criterion, "Which situation seems less impossible?” was then used to help Mr. H rank order the situations. He also expressed concern that "normal" people do not engage in "weird" situations deliberately. He and I discussed the concept that "normal" people might inadvertently engage in exposures without conscious awareness.

During the fourth session, exposures were contextualized within the framework of Mr. H's life goals. If he could "become more normal” by gradually decreasing his need to handwash, this would increase the likelihood of his being able to initiate relationships with famous women and pursue his Hollywood dreams. Every exposure was then framed as being in the 
Values-Focused Exposure and Response Prevention in the Treatment of Comorbid

Schizophrenia and Obsessive-Compulsive Disorder: The Case of "Mr. H"

A. Golden and R. M. Holaway

Pragmatic Case Studies in Psychotherapy, http://pcsp.libraries.rutgers.edu

Volume 9, Module 1, Article 3, pp. 65-80, 02-17-13 [copyright by authors]

service of his life goals, with Mr. $\mathrm{H}$ and I both agreeing before every exposure that if $\mathrm{Mr} . \mathrm{H}$ could become "normal", he would also have a higher probability of forming relationships with famous actresses and pursuing a film career in Hollywood.

In the fourth session, Mr. $\mathrm{H}$ was able to pick an object off the floor, then wait 20 seconds before washing his hands. He initially denounced the exposure exercise as being "impossible" for him to perform. I asked whether he would be willing to try to the exercise for 30 seconds; he responded, "No." I then asked whether he would be willing to try the exercise for 30 seconds with the knowledge that he could stop at any time if he felt overwhelmed. He agreed and was able to complete the exercise as it had been originally formulated.

In the fifth session, Mr. $\mathrm{H}$ held a pair of sunglasses that I had tossed on the floor for as long as he could (20 minutes). As in the fourth session, he initially labeled this exercise as "impossible" and also "embarrassing." I used the same strategy of asking whether he would be willing to engage in the exercise for 30 seconds with the understanding that he could escape at any time if he became extremely distressed, to which he agreed. He was ultimately able to hold the sunglasses for 20 minutes.

In line with the above, subsequent sessions began with the following sequence: my reminding $\mathrm{Mr}$. $\mathrm{H}$ of the exposure hierarchy exercise in which he had chosen to engage during that session; Mr. H expressing hesitation or complete resistance due to the exercise seeming "impossible" or "embarrassing;" my offering to reduce the time of the exposure or to allow Mr. $\mathrm{H}$ to cease the exposure if he felt overwhelmed; and $\mathrm{Mr}$. $\mathrm{H}$ eventually completing the initially agreed-upon exposure without further visible ambivalence.

During the sixth session, Mr. $\mathrm{H}$ was able to place a pair of sunglasses that had been on the floor on his head for five minutes and delay hand-washing by five minutes.

Mr. $\mathrm{H}$ missed the seventh session due to a miscommunication between him and his board-and-care operator, Ms. O.

The eighth session involved Mr. H eating a cracker that the therapist had placed on the floor. He did not ask to wash his hands at all, and admitted that he felt "comfortable" after the exposure.

During the ninth session, Mr. $\mathrm{H}$ flushed a toilet and delayed hand-washing past the end of the session. He reported that he was using the bathroom at his home less often for the purpose of washing his hands.

At the beginning of the tenth session, Mr. $\mathrm{H}$ continued to report that he was washing his hands less at home. He also demonstrated more optimism, stating that if he wanted to improve, he would have to "try hard," rather than insisting that improving his behaviors was impossible. He was able to delay hand-washing after picking up unused toilet paper from the floor and after picking up the therapist's watch from a garbage can. 
Values-Focused Exposure and Response Prevention in the Treatment of Comorbid

Schizophrenia and Obsessive-Compulsive Disorder: The Case of "Mr. H"

A. Golden and R. M. Holaway

Pragmatic Case Studies in Psychotherapy, http://pcsp.libraries.rutgers.edu

Volume 9, Module 1, Article 3, pp. 65-80, 02-17-13 [copyright by authors]

At the beginning of the eleventh session, Mr. H again stated that he would have to "work hard" if he wanted to get better. However, Mr. H expressed that he was "not normal," because a "normal” person would be able to pick up hairpins off the floor for a famous actress. The clinician asked Mr. H whether he would be afraid to pick hairpins off the floor for any woman, such as the therapist herself, another female mental health professional at the clinic, Mr. H's board-and-care operator, Ms. O, etc. Mr. H firmly answered "no" to these questions, insisting that he would only be afraid to pick up hairpins for a particular famous actress. I asked Mr. H's permission to assume the role of the famous actress in the upcoming exposure exercise. $\mathrm{Mr}$. $\mathrm{H}$ expressed his understanding that I was "pretending” to be the famous actress and was not actually the famous actress. Mr. $\mathrm{H}$ also expressed that he was still anxious about picking up the hairpins, because I was going to "pretend to be like" the famous actress.

I role-played the famous actress who required that Mr. H pick up approximately 20 hairpins from the bathroom floor. At the end of the session, he thanked me for helping him to "become normal."

Session twelve involved Mr. H sitting on five pieces of paper that the therapist had placed on the floor. The session concluded with a review of Mr. H's successes and their contributions to the progress of treatment.

At the beginning of treatment, I obtained Mr. H's consent to speak with his board-andcare operator, Ms. O, about his treatment. Ms. O was eager to become involved in facilitating Mr. H's homework assignments. Ms. O, Mr. H., and I agreed that Ms. O would strive to drop a small object, such as her sunglasses, onto the floor five times each day and ask Mr. $\mathrm{H}$ to retrieve it. I checked in regularly with Ms. O, who reported that at the beginning of treatment, $\mathrm{Mr}$. $\mathrm{H}$ refused to pick up the small objects that she had tossed onto the floor, but that by the end of treatment, Mr. H was “almost annoyed” by her requests, as he viewed them as brief obnoxious interruptions of his activities. She reported that he retrieved the objects as requested, but then quickly returned to whatever activity in which he was presently engaged without having to handwash.

\section{THERAPY MONITORING AND USE OF FEEDBACK INFORMATION}

Due to Mr. H's severe level of agitation, it was not possible for me to use standardized questionnaires or rating scales such as the Yale-Brown Obsessive Compulsive Scale (Y-BOCS; (Goodman, Price, Rasmussen, Mazure, Delgado, et al., 1989; Goodman, Price, Rasmussen, Mazure, Fleischman, et al., 1989); the Maudsley Obsessional-Compulsive Inventory (MOCI; Hodgson \& Rachman, 1977); or the Padua Inventory (Sanavio, 1988).

Instead, each week, I met with my supervisor, the second author, to monitor my adherence to ERP standards, Mr. H's compliance with treatment, the efficacy of the treatment, and any obstacles to treatment. My supervisor and I also problem-solved logistical issues, such as gaining access to the clinic's restroom during Mr. H's scheduled session times to conduct exposures. 


\section{CONCLUDING EVALUATION OF THE THERAPY'S PROCESS AND OUTCOME}

By the end of the twelfth session, Ms. O, Mr. H’s board-and-care operator, remarked that there was a "big difference" in Mr. H's behavior. Prior to treatment, he had lined up for his board-and-care home's restroom faucet every half hour; after treatment, he was "not lining up at all.” Before treatment, he had insisted on washing his walker upon entering the board-and-care home; after treatment, he only washed it once per day. At a recent visit to his audiologist's office, Mr. H had "no reaction" to his audiologist handling his hearing aids and handing them back to him; before treatment, he would have been "freaked out." Ms. O excitedly reported that she had chaperoned $\mathrm{Mr}$. $\mathrm{H}$ and other residents on a road trip to a seaside village. $\mathrm{Mr}$. $\mathrm{H}$ had not asked once to stop at a public restroom to wash his hands on the journey there or back, whereas on previous trips to the same destination, he had perseverated in his requests.

Mr. H's observations about his own obsessions and compulsions fluctuated in their tone. Within the course of one session, he would state optimistically that if he wished to get better, he would have to work hard, and thanked me for helping him become "normal"; at another point, he would lament that he was "the same." While his insight into the objective decrease in his own compulsive behaviors may have been limited, he did admit that he was fastening his seatbelt by himself, an observation that was corroborated by Ms. O. She said that while in the past he had struggled and complained for 5 minutes that he was frustrated while the other residents waited in the van, he had simply fastened his seat belt without a sound on the group's recent road trip.

Ms. O added that she had noticed a decrease in various helpless behaviors along with compulsions. Perhaps, having demonstrated to himself that he could expose himself to feared stimuli in the service of his ultimate goal, Mr. $\mathrm{H}$ had acquired some sense of mastery. During multiple sessions, Mr. H expressed the concern that he was not normal because he could not engage in a certain behavior, then proved to himself by the end of the session that he could indeed engage in the behavior. At the end of one session, he even thanked me for helping him to become more "normal." The treatment team believes that Mr. H's pattern throughout therapy of insisting that he was "not normal" at the beginning of a session but then proving to himself experientially that he could engage in a "normal" behavior by the end of a session, along with the therapist's summarizing this pattern for Mr. H toward the end of treatment, contributed to this sense of mastery. Grayson (2003, p. 71) underscores the sense of mastery that can be acquired through ERP.

Exposure and response prevention helps you with this problem [feeling helpless and hopeless in the face of your obsessions] by teaching you to respond to fears in a new way: by confronting and overcoming them. As treatment begins to take hold, exposure becomes a response to not only fight OCD, but one that inspires hope and competence.

Turkington, Kingdon, and Chadwick (2003, p. 98) explain that

Schizophrenia as a diagnosis pulls together a group of people who vary considerably in symptom presentation and course, number of hospital admissions, self-esteem, depression, suicidality, social/family support, response to medication, etc. 
Values-Focused Exposure and Response Prevention in the Treatment of Comorbid

Schizophrenia and Obsessive-Compulsive Disorder: The Case of "Mr. H"

A. Golden and R. M. Holaway

Pragmatic Case Studies in Psychotherapy, http://pcsp.libraries.rutgers.edu

Volume 9, Module 1, Article 3, pp. 65-80, 02-17-13 [copyright by authors]

Despite this diversity, Turkington, Kingdon, and Weiden (2006) report that the current evidence for cognitive-behavioral therapy (CBT) for schizophrenia alone is so strong that it is now accepted as part of the evidence-based treatment in the United Kingdom for the medicationresistant form of the condition. Key techniques involve developing a therapeutic alliance based on the patient's perspective, generating alternative explanations for positive symptoms, and minimizing the severity and distress from the symptoms (Turkington et al., 2006).

But the question remains: How do we apply CBT for schizophrenia to individuals experiencing the disorder along with OCD, particularly when the patient is more distressed by his obsessions and compulsions than by the content of his delusion-and who may, in fact, be motivated to overcome his OCD symptoms in the service of the content of his delusion? Little has been written about the implementation of CBT for patients with disorders comorbid with schizophrenia.

Other issues include how to determine the best practice for addressing comorbid schizophrenia and OCD when the patient is too agitated to engage in CBT to address the content of positive symptoms through guided discovery, as would have been the case with $\mathrm{Mr}$. H. Mr. $\mathrm{H}$ was able to engage in exposure sessions for a maximum of 20 minutes, at which point he would begin to express great pessimism, making statements such as, "This is too embarrassing," "This is impossible," and "I need more medication," becoming restless and insisting on a premature conclusion to the session.

Given that CBT for schizophrenia is contraindicated for patients who are "too agitated" (Turkington et al., 2006, p. 369), a purely behavioral approach, rather than one combining both guided discovery and exposures may be more effective, especially when the disorder is comorbid with OCD. In individuals with schizophrenia, the presence of comorbid OCD or obsessivecompulsive symptoms has been associated with a poorer prognosis (Fenton \& McGlashan, 1986; Berman, Kalinowski, Berman, Lengua, \& Green, 1995; Poyurovsky, Weizman, \& Weizman, 2004); a significantly higher risk of suicide attempt (Sevincok, Akoglu, \& Kokcu, 2007); and lower functioning levels (Hwang, Morgan, \& Losconzcy, 2000). However, the outcome is likely to improve when the OCD is recognized and treated (Gangdev, 2002).

Hagen and Nordahl (2008), using a case study, demonstrated that an individual with paranoid schizophrenia is capable of engaging in behavioral experiments. In the case of the patient described, the therapist was able to help the patient design situations to generate evidence to test the reality of his delusions. The authors reported that the patient is now enrolled in university and has not relapsed. Research shows that behavioral strategies engender cognitive, emotional, and behavioral change (Rouf, Fennel, Westbrook, Cooper, \& Bennett-Levy, 2005). The case of Mr. H reveals that after an individual with comorbid schizophrenia and OCD has engaged in a values-focused behavioral treatment (ERP), he may feel a sense of mastery and exhibit less helpless behaviors, feel less stressed by a fear of contamination and need to wash, and spend less time hand-washing.

Some of Mr. H’s original mental health care workers originally presumed that his delusions would need to be addressed successfully prior to his being able to engage in an 
Values-Focused Exposure and Response Prevention in the Treatment of Comorbid

Schizophrenia and Obsessive-Compulsive Disorder: The Case of "Mr. H"

A. Golden and R.M. Holaway

Pragmatic Case Studies in Psychotherapy, http://pcsp.libraries.rutgers.edu

Volume 9, Module 1, Article 3, pp. 65-80, 02-17-13 [copyright by authors]

effective course of ERP. Mr. H had been seen at the same clinic over the course of several decades, and his most recent mental health care worker transferred his care to us specifically for the treatment of his obsessions and compulsions. Mr. H was seen concurrently by a staff psychiatrist for medication management of his psychotic symptoms. In consulting with the staff psychiatrist, we learned that Mr. H was on the "maximum" dosage of his antipsychotic medications and was still experiencing psychotic delusions. Since (1) Mr. H’s referring psychologist suggested that he would benefit most from a focus on his obsessions and compulsions, (2) Mr. H’s delusions were longstanding and appeared medication-resistant, and (3) Mr. H's obsessions and compulsions seemed more distressing than his delusions, the psychological treatment could proceed with a focus on helping $\mathrm{Mr}$. $\mathrm{H}$ to address his OCD primarily. Mr. H's case demonstrates that it is not necessary to ameliorate delusions prior to addressing obsessions and compulsions with a behavioral treatment (ERP). Thus, ERP tailored to the needs of the individual patient may very well be effective for patients with OCD and psychosis.

While the treatment of an agitated patient with schizophrenia and OCD in the current case study did not involve challenging the patient's delusions, it did share a key principle with CBT for schizophrenia alone: the development of a therapeutic alliance based on the patient's perspective (Turkington et al., 2006). This involves empathy, respect, and unconditional positive regard for the patient (Turkington et al., 2006). In line with this, l always asked $\mathrm{Mr}$. H whether he would care for a cup of coffee at the beginning of his visit, as he had expressed that this was his favorite beverage but that it was often difficult for him to fetch because he had to place both hands on his walker to travel. He was always greatly appreciative when I made this gesture acknowledging his wishes. CBT for schizophrenia emphasizes the "subjective and connections among the patient's beliefs, feelings, and actions, irrespective of whether these beliefs are reality based” (Turkington et al., p. 366). Sometimes during a session, Mr. H would lose focus on the exposure task at hand and begin a narration based on his positive symptoms. At these times I empathized with the feelings generated by Mr. H's beliefs and strove to cultivate understanding for his perceived experience rather than harbor silent judgment.

Some opponents of this approach might argue that it is unfair to the patient to equate a delusion, by definition a false belief, with a core value in the sense of ACT theory, as described above in the Guiding Conception section. However, as the treatment team we maintained that Mr. H detested the monotony of his life, claiming that he spent every day "smoking and drinking coffee." He had longed for years to go to Hollywood and become a movie star. At the same time, he suffered from time-consuming, ego-dystonic contamination obsessions and compulsions, which he believed were preventing him from first and foremost being "normal," and ultimately from pursuing his dream. We carefully weighed the risks and benefits of equating Mr. H's grandiose delusions with core values. It was ultimately determined that even if $\mathrm{Mr}$. $\mathrm{H}$ did not achieve his aspiration of becoming a movie star, his quality of life would improve markedly subsequent to the elimination or reduction of his obsessions and compulsions. He would no longer confine himself to the proximity of the bathroom sink of his board-and-care home, destined to quench the boredom in between bathroom visits by drinking coffee and smoking an endless chain of cigarettes. This is precisely what happened. By the end of treatment, Mr. $\mathrm{H}$ reported that he was taking himself on occasion to a fast-food restaurant for 
Schizophrenia and Obsessive-Compulsive Disorder: The Case of "Mr. H"

A. Golden and R. M. Holaway

Pragmatic Case Studies in Psychotherapy, http://pcsp.libraries.rutgers.edu

Volume 9, Module 1, Article 3, pp. 65-80, 02-17-13 [copyright by authors]

evening meals. He may not have become a film star, but he gained something almost as important to him: greater independence.

This study suffers from the obvious limitation of a sample size of one, which limits external validity. It will also not always be true that a grandiose delusion contains a more intermediate, realistically achievable value. On the other hand, the addition of a values-based perspective to a traditional cognitive behavioral approach may provide hope, motivation, and healing for some individuals with comorbid OCD and schizophrenia.

In conclusion, there is a subgroup of individuals who demonstrate comorbid diagnoses of schizophrenia and OCD. To target obsessions and compulsions in such individuals, practitioners may need to alter ERP, given the unique features of this subgroup. Providers may approach this treatment from within the context of a patient's delusion after careful consultation involving a consideration of the costs and benefits of such an approach. For patients with co-occurring schizophrenia and OCD, reducing or eliminating obsessions and compulsions through a behavioral treatment may not result in the achievement of a delusional goal or value, but may contribute to an increased sense of mastery.

\section{REFERENCES}

Abramowitz, J. S. (1997). Effectiveness of psychological and pharmacological treatments for obsessive-compulsive disorder: A quantitative review. Journal of Consulting and Clinical Psychology, 65, 44-52.

American Psychiatric Association (1994). Diagnostic and statistical manual of mental health disorders $\left(4^{\text {th }}\right.$ ed). Washington, D.C.

American Psychiatric Association. (2007). Practice guideline for the treatment of patients with obsessive-compulsive disorder. American Journal of Psychiatry, 164 (July supplement).

Bach, P., \& Hayes, S. C. (2002). The use of acceptance and commitment therapy to prevent the rehospitalization of psychotic patients: A randomized controlled trial. Journal of Consulting and Clinical Psychology, 70(5), 1129-1139.

Berman, I., Kalinowski, A., Berman, S. M., Lengua, J., \& Green, A. I. (1995). Obsessive and compulsive symptoms in chronic schizophrenia. Comprehensive Psychiatry, 36, 6-10.

Bloy, S., Oliver, J. E., \& Morris, E. (2011). Using acceptance and commitment therapy with people with psychosis: A case study. Clinical Case Studies 10(5), 347-359.

Clark, R. E., Bartels, S. J., Mellman, T. A., \& Peacock, W. J. (2002). Recent trends in antipsychotic combination therapy of schizophrenia and schizoaffective disorder: Implications for state mental health policy. Schizophrenia Bulletin, 28, 75-84.

Covell, N. H., Jackson, C. T., Evans, A. C., \& Essock, S. M. (2002). Antipsychotic prescribing practices in Connecticut's public mental health system: Rates of changing medications and prescribing styles. Schizophrenia Bulletin, 28, 17-29.

Foa, E. B., Abramowitz, J. S., Franklin, M. E., \& Kozak, M. J. (1999). Feared consequences, fixity of belief, and treatment outcome in patients with obsessive compulsive disorder. Behavior Therapy, 30, 717-724. 
Values-Focused Exposure and Response Prevention in the Treatment of Comorbid

Schizophrenia and Obsessive-Compulsive Disorder: The Case of "Mr. H"

A. Golden and R.M. Holaway

Pragmatic Case Studies in Psychotherapy, http://pcsp.libraries.rutgers.edu

Volume 9, Module 1, Article 3, pp. 65-80, 02-17-13 [copyright by authors]

Foa, E. B., Kozak. M. J., Goodman, W. K., Hollander, E., Jenike, M., \& Rasmussen, S. (1995). DSM-IV field trial: Obsessive-compulsive disorder. American Journal of Psychiatry, 152, 90-96.

Foa, E. B., Steketee, G. S., Grayson, J. B., Turner, R. M., \& Latimer, P. R. (1984). Deliberate exposure and blocking of obsessive-compulsive rituals: Immediate and long term effects. Behaviour Therapy, 15, 450-472.

Franklin, M. E., \& Foa, E. B. (2008). Obsessive-compulsive disorder. In Barlow, D. H. (Ed.), Clinical handbook of psychological disorders: A step-by-step treatment manual, $4^{\text {th }}$ edition, (pp. 164-215). New York: The Guilford Press.

Ganesan, P., Kumar, T. C. R., \& Khanna, S. (2001). Canadian Journal of Psychiatry, 46, 750753.

Gangdev, P. (2002). The relationship between obsessive-compulsive disorder and psychosis. Australasian Psychiatry, 10(4), 405-410.

García, J.M. \& Pérez, M. (2001). ACT as a treatment for psychotic symptoms: The case of auditory hallucinations. Análisis y Modificación de Conducta, 27(113), 455-472.

Gaudiano, B. A., \& Herbert, J. D. (2006). Acute treatment of inpatients with psychotic symptoms using acceptance and commitment therapy: Pilot results. Behaviour Research and Therapy, 44, 415-437.

Goodman, W. K., Price, L. H., Rasmussen, S. A., Mazure, C., Delgado, P., Heninger, G. R., \& Charney, D. S. (1989). The Yale-Brown Obsessive Compulsive Scale II: Validity. Archives of General Psychiatry, 46, 1012-1016.

Goodman, W. K., Price, L. H, Rasmussen, S. A., Mazure, C., Fleischman, R. L., Hill, C. L., Heninger, G. R., \& Charney, D. A. (1989). The Yale-Brown Obsessive Compulsive Scale: 1. Development, use, and reliability. Archives of General Psychiatry, 46, 10061011.

Hagen, R., \& Nordahl, H. M. (2008). Behavioral experiments in the treatment of paranoid schizophrenia: A single case study. Cognitive and Behavioral Practice, 15, 296-305.

Harvard School of Medicine. (2005). National Comorbidity Survey (NCS) and National Comorbidity Survey Replication (NCS-R). Retrieved September 27, 2011, from http://www.hcp.med.harvard.edu/ncs/

Hayes, S. C., Strosahl, K. D., \& Wilson, K. G. (1999). Acceptance and commitment therapy: An experiential approach to behavior change. New York: Guilford Press.

Hodson, R. J., \& Rachman, S. (1977). Obsessional-compulsive complaints. Behaviour Research and Therapy, 15, 389-395.

Hwang, M. Y., Yum, S. Y., Kwon, J. S., \& Opler, L. A. (2005). Management of schizophrenia with obsessive-compulsive disorder. Psychiatric Annals, 35(1), 36-43.

Kozak, M J., \& Foa, E. B. (1994). Obsessions, overvalued ideas, and delusions in obsessivecompulsive disorder. Behaviour Research and Therapy, 32, 343-353.

Leckman, J. F., Denys, D., Simpson, H. B., Mataix-Cols, D., Hollander, E., Saxena, S., Miguel, E. C., Rauch, S. L., Goodman, W. K., Phillips, K. A., \& Stein, D. J. (2010). Obsessivecompulsive disorder: A review of the diagnostic criteria and possible subtypes and dimensional specifiers for DSM-V. Depression \& Anxiety, 27(6), 507-527. 
Values-Focused Exposure and Response Prevention in the Treatment of Comorbid

Schizophrenia and Obsessive-Compulsive Disorder: The Case of "Mr. H"

A. Golden and R.M. Holaway

Pragmatic Case Studies in Psychotherapy, http://pcsp.libraries.rutgers.edu

Volume 9, Module 1, Article 3, pp. 65-80, 02-17-13 [copyright by authors]

McDonough, M., \& Kennedy, N. Pharmacological management of obsessive compulsive disorder: A review for clinicians. Harvard Review of Psychiatry, May/June 2002, 127137.

Mueser, K. T., \& Noordsy, D. L. (2005). Cognitive behavior therapy for psychosis: A call to action. Clinical Psychology: Science and Practice, 12(1), 68-71.

Noordsy, D. L., Mellman, T., Larsen, H., Logothetis, L., \& O’Keefe, C. D. (2001). Outcomes of treatment with multiple antipsychotic agents. Paper presented at the $41^{\text {st }}$ Annual NCDEU Meeting, Scottsdale, AZ.

Owashi, Toshimi; Ota, Arimitsu; Otsubo, Tempei; Susa, Yuko, \& Kamijima, Kunitoshi. 2010). Obsessive-compulsive disorder and obsessive-compulsive symptoms in Japanese inpatients with chronic schizophrenia - A possible schizophrenic subtype. Psychiatry Research, 179(3), 241-246.

Pankey, Julieann. (2003). Acceptance and commitment therapy for psychosis. International Journal of Psychology \& Psychological Therapy, 3(2), 311-328.

Poyurovsky, M., Weizman, A., \& Weizman, R. (2004). Obsessive-compulsive disorder in schizophrenia: Clinical characteristics and treatment. CNS Drugs, 18, 989-1010.

Rodriguez, C. I., Corcoran, C., \& Simpson, H. B. (2010). Diagnosis and treatment of a patient with both psychotic and obsessive-compulsive symptoms. American Journal of Psychiatry, 167, 754-761.

Rouf, K., Fennel, M., Westbrook, D., Cooper, M., \& Bennett-Levy, J. (2005). Devising effective behavioural experiments. In J. Bennett-Levy, G. Butler, M. Fennell, A. Hackmann, M. Mueller, \& D. Westbrook (Eds.), Oxford guide to behavioural experiments in cognitive therapy (pp. 21-59). Oxford: Oxford University Press.

Salkovskis, P. M., \& Campbell, P. (1994). Thought suppression induces intrusion in naturally occurring negative intrusive thoughts. Behaviour Research and Therapy, 32, 1-8.

Sanavio, E. (1988). Obsessions and compulsions: The Padua inventory. Behaviour Research and Therapy, 26, 169-177.

Sevincok, L., Akkoglu, A., \& Kokcu, F. (2007). Suicidality in schizophrenic patients with and without obsessive-compulsive disorder. Schizophrenia Research, 90, 198-202.

Strous, R. D., Patel, J. K., Zimmet, S., \& Green, A. I. (1999). Clozapine and paroxetine in the treatment of schizophrenia with obsessive-compulsive features. The American Journal of Psychiatry, 156(6), 973-974.

Turkington, D., Kingdon, D., \& Chadwick, P. (2003). Cognitive-behavioural therapy for schizophrenia: Filling the therapeutic vacuum. British Journal of Psychiatry, 183,98-99.

Turkington, D., Kingdon, D., \& Weiden, P. J. (2006). Cognitive behavior therapy in schizophrenia. American Journal of Psychiatry, 163(3), 365-373.

van Balkom, A. J. L. M., van Oppen, P., Vermeulen, A. W. A., van Dyck, R., Nauta, M. C. E., \& Vorst, H. C. M. (1994). A meta-analysis on the treatment of obsessive-compulsive disorder: A comparison of antidepressants, behavior, and cognitive therapy. Clinical Psychology Review, 14, 359-381

Wegner, D. M., Schneider, D. J., Carter, S. R., \& White, T. L. (1987). Paradoxical effects of thought suppression. Journal of Personality and Social Psychology, 53, 5-13. 